\title{
Impacts de l'élévation du niveau de la mer sur l'évolution future d'une côte basse à lagune de la péninsule du Cap Bon (Nord-Est de la Tunisie): approche cartographique
}

\author{
Noura BRAHMI ${ }^{\mathrm{a}, \mathrm{c}} ;$ Mohsen DHIEB $^{\mathrm{b}, \mathrm{c}}$ et Mohamed Chedly RABIA ${ }^{\mathrm{a}}$ \\ ${ }^{a}$ Unité de Recherche Géomatique des Géosystèmes, Faculté des Lettres des Arts et des Humanités de la Mannouba, Université de \\ Mannouba.E-mail:noura_brahmi@yahoo.fr;Mohame.Rabiamch@flm.rnu.tn. \\ ${ }^{b}$ Laboratoire SYFACTE, Faculté des Lettres et des Sciences Humaines de Sfax (détaché auprès de l'Université du roi Abdulaziz, \\ Arabie Saoudite),Université de Sfax. / mohsendhieb2003@yahoo.fr. \\ ${ }^{c}$ Association Tunisienne de la Cartographie et de l'Information Géographique (ATCIG).
}

\begin{abstract}
La submersion marine est l'une des principales menaces qui pèsent sur les zones humides littorales de la péninsule du Cap Bon. Or, cette menace est amenée à se renforcer en raison du réchauffement climatique qui entraînera une élévation du niveau marin et vraisemblablement un renforcement de l'intensité des tempêtes et des cyclones tropicaux d'ici à l'horizon 2100. L'objectif a donc été d'évaluer la vulnérabilité de la lagune face à la submersion. Dans cette optique, après avoir identifié les enjeux liés à l'élévation du niveau de la mer pour la lagune, nous avons dressé une cartographie prévisionnelle des risques de submersion par l'intermédiaire d'une application cartographique basée sur une modélisation numérique de terrain et analysé les impacts potentiels de ce phénomène. La cartographie est donc amenée à devenir centrale pour l'étude de l'impact du risque de la submersion marine sur les lagunes côtières et la gestion de ces espaces dans les décennies à venir. La cartographie de l'aléa submersion marine a montré que l'ouverture épisodique de brèches lors des tempêtes pourrait entraîner la submersion de l'ensemble du domaine lagunaire et aurait un impact morphogénique essentiel. En effet, le phénomène d'une accélération de l'élévation du niveau marin et d'un renforcement des tempêtes génère le morcellement du cordon littoral qui sépare la lagune de la mer. Par ailleurs, les pertes de matériel sédimentaire pour la plage augmenteront, dans la mesure où, lors d'une tempête, une grande partie des matériaux déplacés par les vagues dans les étangs par submersion ou par ouverture d'une brèche, ne peut être récupérée par la suite. Tout ceci constitue un facteur d'accélération du recul ou de disparition de la plage déjà très érodée. Les impacts de cette submersion pourraient être importants en absence de mesures préventives. Elle aurait ainsi des répercussions profondes sur les systèmes naturels et environnementaux et sur la qualité de vie de la population locale.
\end{abstract}

Keywords: Cartographie, modèle numérique de terrain, submersion marine, vulnérabilité, lagune.

\section{Introduction}

L'impact potentiel des changements climatiques sur les marges des calottes Antarctiques et du Groenland pourraient causer une élévation du niveau marin. Des études récentes suggèrent également que l'élévation du niveau marin pourrait avoir des conséquences importantes sur les zones humides côtières. Par ailleurs, les conséquences de l'élévation du niveau marin sur les systèmes côtiers dépendent fortement de la géomorphologie côtière, mais ils doivent être analysés au sein de $1^{\prime}$ ensemble des phénomènes pouvant avoir un effet sur la morphodynamique littorale, notamment les événements extrêmes. Pour cartographier les impacts d'une potentielle élévation du niveau marin sur les côtes basses à lagune, il est nécessaire de mettre en place une base de données géocodée qui intègre toutes les paramètres physique, environnementale, et socioéconomique qui gouvernent la morphodynamique de ces côtes.

Dans ce papier, d'une part il s'agissait d'identifier et estimer des indicateurs de vulnérabilité à l'érosion et à la submersion marine pour les côtes à lagune, face au changement climatique à échéance des années 2100 . D'autre part, le rôle aggravant que peut avoir le facteur d'occupation humaine du littoral sur cette vulnérabilité a été étudié. Et enfin, l'impact de l'aléa submersion marine sur cette côte basse en général et sur la lagune en particulier. Cette recherche est basée sur l'étude d'une lagune qui se situe dans une côte sableuse basse fortement anthropisée, dans la quelle on trouve une lagune temporaire, celle de Kélibia. La lagune de Kélibia se situe sur la pointe nord-est de la péninsule du Cap Bon, se présentait dans le temps comme une seule entité couvrant une zone humide d'environ 60 ha. Aujourd'hui cette lagune est divisée par une route en deux sous-entités (Fig.1). Le site est vu par la population locale comme répulsif et d'ailleurs c'est le cas de tous les milieux côtiers humides, parce que sa végétation halophile abrite des espèces de moustiques nuisibles pour les habitants riverains de ces sites. C'est pour cela qu'ils ont servi comme lieu de décharge. La Sebkhet Kélibia a été partiellement remblayée et envahie par le bâti et se trouve aujourd'hui entourée de tous les côtés par les quartiers urbains. La superficie de la lagune se limite actuellement à 16 ha seulement. La diversité et le nombre des activités 
humaines qui se développent aux voisinages de cette zone humide lui confèrent une vulnérabilité d'un niveau et d'une nature spécifiques.

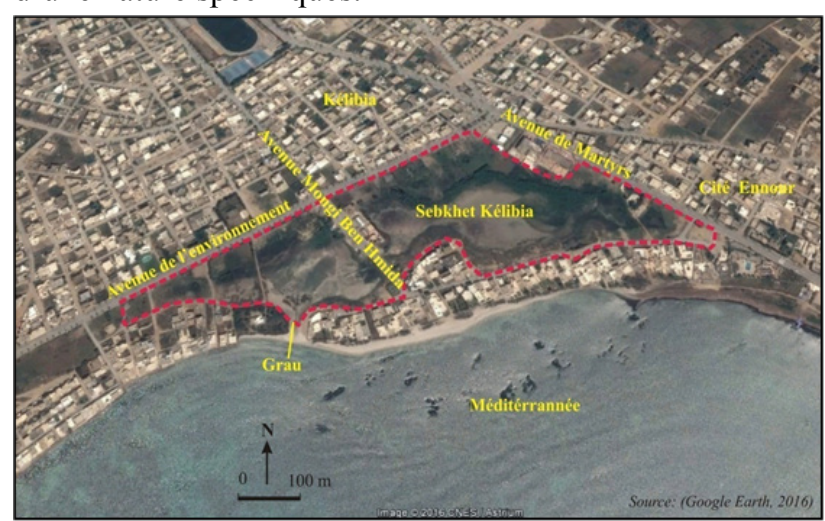

Fig. 1. Localisation de la Sebkhet Kélibia. Le tireté rouge matérialise les limites de la lagune.

\section{Méthodologie de la cartographie du risque submersion marine}

Nous proposons dans ce paragraphe de mettre en place une méthodologie de définition des zones submersibles à partir d'une modélisation numérique du terrain. La méthodologie repose, d'une part, sur la création d'un référentiel topographique fin du site et d'autre part, sur la détermination des niveaux d'eau susceptibles d'être atteints à la fois en fonction des surcotes éventuelles, et des valeurs d'élévation du niveau marin prévues pour la fin du 21ème siècle. La méthode suivie s'articule généralement autour de trois points (Cariolet, 2011), (Cariolet et al. 2012). Elle consiste dans un premier temps à estimer l'aléa submersion en quantifiant les niveaux d'eau extrêmes à la côte pour une période de retour donnée (généralement de 100 ans). Cette estimation tient compte des différents paramètres agissant sur la déformation du plan d'eau à la côte, notamment la surcote (élévation due à une baisse de la pression atmosphérique et action des vents) et l'élévation de l'eau à la côte due à l'agitation marine. Dans un second temps, cette hauteur d'eau est superposée à la topographie du lieu étudié afin de délimiter la zone potentiellement submersible. L'estimation du risque est enfin réalisée en tenant compte des éléments socio-économiques et environnementaux directement menacés (Solomon and Forbes, 1999 ; Meur-Férec et al. 2008 ; De Pippo et al., 2008 ; Vinchon et al., 2009 cités par Cariolet et al., 2012). Cette démarche constitue le socle méthodologique pour la cartographie du risque de submersion réalisée au niveau de la côte basse à lagune. L'impact des surcotes correspondantes en termes de submersion a été estimé à l'aide de modèles numériques de terrain (MNT). Ces MNT ont été construits par compilation des différentes données topographiques au format numérique sous le logiciel Arc gis. Après avoir identifié les enjeux liés à l'élévation du niveau de la mer, nous avons dressé une cartographie prévisionnelle des risques de submersion et analysé les impacts potentiels de ce phénomène.

\section{Les enjeux liés à l'élévation du niveau marin pour la lagune de Kélibia}

La menace de l'élévation du niveau de la mer pèse essentiellement sur la survie du site et pose des véritables problèmes de gestion plusieurs grands enjeux. A partir des cartes d'occupation des sols, des cartes topographiques (1/25000), des plans de gestion de la Sebkhet Kélibia, des images Google Earth et des travaux de terrain, les enjeux ont été vectorisés sous Arc Gis. Ces espaces à risque sont variés et étendus: construction de front de mer, plages sableuses, ouvrages de protection, infrastructure hôtelière, infrastructure routière, biodiversité...etc. Notre objectif est d'essayer de comprendre les modifications qui pourraient résulter suite à une submersion marine et surtout les pertes susceptibles de se produire en considérant les différents scénarios d'élévation du niveau marin considérés. Les enjeux peuvent être regroupés en deux principales catégories : enjeux environnementaux et enjeux socio-économiques.

\subsection{Enjeux environnementaux:}

La plage, les limites du Domaine Public Maritime, la biodiversité et les legs d'un cordon dunaires fortement anthropisé constituent les enjeux environnementaux les plus menacés par la montée du niveau de la mer. L'érosion marine constitue la menace la plus importante. En effet, elle est favorisée par des facteurs naturels et accélérée par les interventions humaines imprévisibles (aménagement du front de mer, rejets terrestres). L'enjeu est également écologique. En effet, en cas d'élévation du niveau de la mer, la submersion éventuelle de la Sebkhet Kélibia risquerait d'entraîner la disparition la végétation halophile qui représentait et représente encore un relais pour certaines espèces d'oiseaux migrateurs protégées en Méditerranée. Cet écosystème humide côtier sera menacé par les changements des conditions de vie liées à la pénétration des eaux marines.

\subsection{Les enjeux socio-économiques}

Pour faire apparaître les enjeux (humains, économiques...) de la zone, il suffit de superposer le M.N.T. avec la carte d'occupation des sols. Les espaces à risque sont variés et étendus : construction de front de mer, des routes plages sableuses, hôtels, des écoles, cafés, restaurants, espaces récréatifs, des ouvrages de protection contre l'érosion ...etc. L'objectif est d'essayer de comprendre les modifications qui pourraient résulter suite à une élévation du niveau marin et surtout les pertes susceptibles de se produire en considérant les différents scénarios. En plus des effets directs sur la morphologie $\mathrm{du}$ cordon littoral, l'élévation du niveau marin aura des conséquences indirectes sur de nombreux secteurs sur la population et l'économie du secteur étudié. Il s'agit essentiellement des secteurs suivants: la population concentrée sur la frange littorale, les infrastructures hôtelières, l'infrastructure routière...etc. L'enjeu est aussi économique. Il concerne la stratégie de défense à adopter face à l'élévation du niveau de la mer. Doit-on opter pour une défense lourde par des brise-lames, des épis et des 
enrochements, avec les coûts importants ou pour une défense plus douce (Anselme et al. 2008).

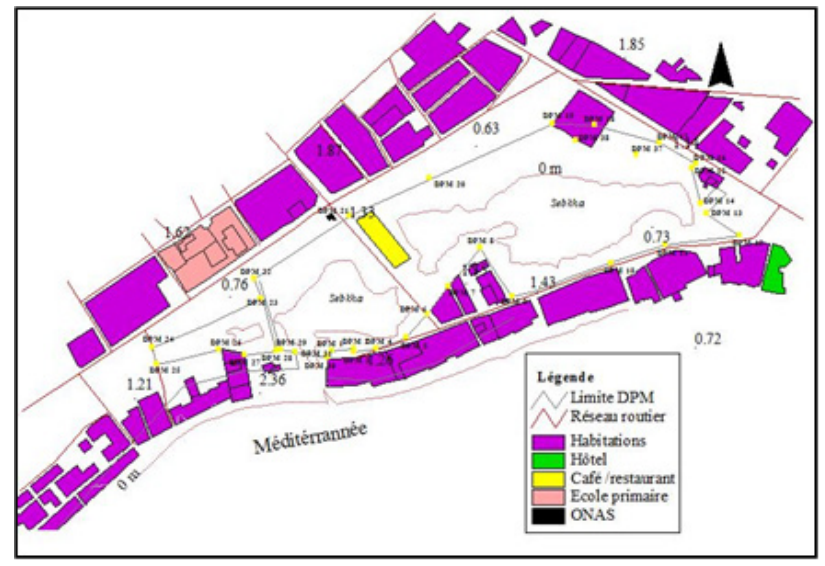

Fig.2. Les enjeux sensibles à une élévation du niveau marin sur la côte à lagune de Kélibia.

\section{Scénarios de submersion marine dans la lagune de Kélibia}

Les hypothèses d'élévation du niveau de la mer pour la Tunisie sont de $0.38 \mathrm{~m}$ pour le scénario minimum de risque, $0.50 \mathrm{~m}$ pour le scénario volontariste et de $0.55 \mathrm{~m}$ pour le scénario de maximum de risque (GIEC, 2007). Le niveau de référence altimétrique considéré est le zéro du Nivellement Général de la Tunisie (NGT).

4.1. Cartographie de la submersion marine de la lagune de Kélibia: situation sans événement exceptionnelle

Les niveaux d'eau susceptibles d'être atteints à la côte de Kélibia dépendent en premier lieu des hauteurs prévues pour la marée astronomique. Celles-ci sont calculées pour le golfe de Hammamet, au niveau des côtes de Sousse, pendant l'année 1976-1977 (LCHF, 1978). D'après ces enregistrements marégraphiques, la marée au Golfe de Hammamet est de type semi-diurne dont l'amplitude moyenne annuelle est de l'ordre de 0,45 m et l'amplitude moyenne mensuelle est comprise entre $0,23 \mathrm{~m}$ et $0,17 \mathrm{~m}$. L'amplitude maximale journalière observée pour 1976 a été de $0,45 \mathrm{~m}$. Les amplitudes minimales exceptionnelles sont de l'ordre de 0,05 à $0,10 \mathrm{~m}$. La côte maximale mesurée en 1976 a été de $+0,58$ m NGT et la côte minimale a été de $-0,41 \mathrm{~m}$ NGT. Ainsi, la connaissance des différents types des niveaux est indispensable pour pouvoir étudier le degré de vulnérabilité et l'ampleur des impacts potentiels de l'élévation accélérée du niveau de la mer. Les caractéristiques hydrographiques du golfe de Hammamet ont été identifiées par I.H.E. (2000 ; Ingénierie de l'Hydraulique et de l'Environnement) comme suit : (tableau 1).

\begin{tabular}{|l|l|} 
& Valeur \\
\hline $\begin{array}{l}\text { Niveau moyen annuel } \\
\begin{array}{l}\text { Niveau maximal des vives eaux } \\
\text { ordinaires }\end{array}\end{array}$ & 0,00 \\
\hline $\begin{array}{l}\text { Niveau maximal des vives eaux } \\
\text { extraordinaires }\end{array}$ & 0,23 \\
\hline $\begin{array}{l}\text { Le niveau maximal } \\
\text { exceptionnel dû à des grandes } \\
\text { surcotes induites par le vent }\end{array}$ & 0,83 \\
\hline
\end{tabular}

Niveau maximal exceptionnel 0,93 dû à des grandes surcotes induites par des coups exceptionnels de vent

Tableau 1 : Caractéristiques hydrographiques actuelles du Golfe de Hammamet en "m NGT" (Source : IHE, 2000).

Dans la côte de Kélibia, les houles dominantes sont généralement de la direction Est. Le manque d'informations sur le régime des houles conduit à prévoir les houles pour le large. Les caractéristiques de la houle dans le Golfe de Hammamet, estimées par "Hidrotécnica Portuguesa" en 1996 (APAL/MEAT, 2001) en fonction de la période de retour, sont indiquées dans le tableau 2. Le tableau montre que la hauteur maximale calculée de la houle au large, varie de $3,1 \mathrm{~m}$ pour une houle annuelle à $6,9 \mathrm{~m}$ pour une houle centennale, alors que la période varie de $7,1 \mathrm{~s}$ à $13,2 \mathrm{~s}$ pour les mêmes périodes de retour. Notons que les hauteurs des houles sont calculées au large où la profondeur d'eau est importante. Près des côtes, par contre, la profondeur devient faible à cause du phénomène de déferlement et les hauteurs des houles subissent des réductions importantes.

\begin{tabular}{l|l|l|l|l|l|l}
$\begin{array}{l}\text { Période de } \\
\text { retour (ans) }\end{array}$ & 1 & 5 & 10 & 25 & 50 & 100 \\
\hline $\begin{array}{l}\text { Hauteur } \\
\text { significative } \\
\text { maximale } \\
\text { (m) }\end{array}$ & 3,1 & 4,3 & 4,9 & 5,7 & 6,3 & 6,9 \\
\hline $\begin{array}{l}\text { Période de } \\
\text { puissance } \\
\text { (secondes) }\end{array}$ & 7,1 & 9,4 & 10,3 & 11,5 & 12,4 & 13,2 \\
\hline
\end{tabular}

Tableau 2 : Caractéristiques de la houle du Golfe de Hammamet (APAL/MEAT, 2001).

Les niveaux marins permanents, récurrents et exceptionnels de la côte du Golfe de Hammamet, selon les différents scénarios de l'IPCC ont été identifiés par I.H.E. (2000) dans le tableau 3. Nous essayerons d'introduire les données des différents scénarios adoptés, sur la base des données fournies par la communication initiale de la Tunisie à la Convention Cadre des Nations Unies sur les Changements Climatiques (C.C.N.U.C.C., octobre 2001) dans le Modèle numérique de terrain simulé.

L'intérêt de la modélisation numérique pour la délimitation des zones submersibles était la possibilité d'inclure le niveau marin extrême et définir ainsi un seuil où la zone pourrait être inondée. Il faut bien évidemment que le référentiel soit le même pour les points levés et le niveau marin extrême (N.G.T). Ceci nous a permis donc de faire apparaître, en dégradation du bleu, les zones submersibles.

$$
\begin{array}{|l|l|l}
\text { Degré } & \text { Etat } & \text { Etat futur à l'horizon } 2100
\end{array}
$$




\begin{tabular}{|c|c|c|c|c|c|}
\hline & $\begin{array}{c}\text { de } \\
\text { fréque } \\
\text { nce }\end{array}$ & $\begin{array}{c}\text { actu } \\
\text { el }\end{array}$ & $\begin{array}{c}\text { Scénario } \\
\text { volontari } \\
\text { ste }\end{array}$ & $\begin{array}{l}\text { Scénar } \\
\text { io de } \\
\text { référen } \\
\text { ce }\end{array}$ & $\begin{array}{c}\text { Scénari } \\
\quad \text { o } \\
\text { maxim } \\
\text { um de } \\
\text { risque }\end{array}$ \\
\hline & & & $38 \mathrm{~cm}$ & $50 \mathrm{~cm}$ & $55 \mathrm{~cm}$ \\
\hline $\begin{array}{l}\text { Niveau } \\
\text { moyen } \\
\text { annuel }\end{array}$ & $\begin{array}{l}\text { Niveau } \\
\text { courant }\end{array}$ & 0,00 & 0,38 & 0,50 & 0,55 \\
\hline $\begin{array}{c}\text { Niveau } \\
\text { maximal } \\
\text { des vives } \\
\text { eaux } \\
\text { ordinaires }\end{array}$ & $\begin{array}{c}2 \\
\text { fois/mo } \\
\text { is }\end{array}$ & 0,15 & 0,53 & 0,65 & 0,70 \\
\hline $\begin{array}{c}\text { Niveau } \\
\text { maximal } \\
\text { des vives } \\
\text { eaux } \\
\text { extraordina } \\
\text { ires }\end{array}$ & $\begin{array}{c}2 \\
\text { fois/ans }\end{array}$ & 0,23 & 0,61 & 0,73 & 0,78 \\
\hline $\begin{array}{c}\text { Niveau } \\
\text { maximal } \\
\text { exceptionne } \\
\text { I du à des } \\
\text { grandes } \\
\text { surcotes } \\
\text { induites par } \\
\text { le vent }\end{array}$ & $\begin{array}{c}1 \\
\text { fois/10 } \\
\text { ans }\end{array}$ & 0,83 & 1,21 & 1,33 & 1,38 \\
\hline $\begin{array}{c}\text { Niveau } \\
\text { maximal } \\
\text { exceptionne } \\
\text { I du à des } \\
\text { grandes } \\
\text { surcotes } \\
\text { induites par } \\
\text { des coups } \\
\text { exceptionne } \\
\text { Is de vent }\end{array}$ & $\begin{array}{c}1 \\
\text { fois/50a } \\
\text { ns }\end{array}$ & 0,93 & 1,31 & 1,43 & 1,48 \\
\hline
\end{tabular}

Tableau 1. Niveaux marins caractéristiques futurs dans le Golfe de Hammamet (m NGT) (Source : I.H.E., 2000).

La submersion de la lagune de Kélibia ne serait possible dans le premier scénario (situation sans tempête) que dans un seul cas ; il faudrait que le niveau de la mer dépasse le niveau maximal des vives eaux ordinaires et à travers la passe actuelle encore ouverte, sans ouverture d'autres brèches dans le cordon littoral (Fig. 2). Les superficies submersibles de la lagune sont présentées dans le tableau 3.

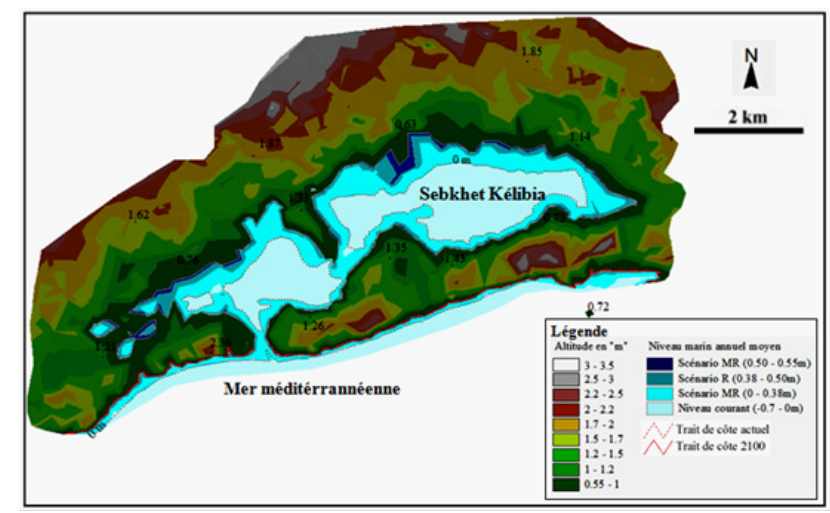

Fig. 2 : Les surfaces inondables dans la lagune côtière de Kélibia selon le niveau marin annuel moyen à l'horizon 2100 (Source : Lèves topographiques par GPS; Scénarios de l'IPCC, 1997; IHE, 2000).

\begin{tabular}{l|l|l|l|l} 
& $\begin{array}{l}\text { Etat } \\
\text { actuel }\end{array}$ & SC_V & SC_R & SC_MR \\
\hline $\begin{array}{l}\text { Superficie } \\
\text { actuelle (ha) }\end{array}$ & 13.27 & 16.98 & 18.18 & 18.80 \\
\hline $\begin{array}{l}\text { Superficie } \\
\text { ajoutée (ha) }\end{array}$ & - & 3.71 & 1.2 & 0.62 \\
\hline
\end{tabular}

Tableau 3: Les superficies submersibles à Sebkhet Kélibia en considérant le niveau annuel moyen (Source : Calculs personnels).

4.1 Cartographie de l'aléa submersion marine à la lagune de Kélibia: situation avec événement exceptionnel

La submersion marine dans ce cas est liée à une élévation du plan d'eau due à la combinaison de processus physiques liés à des phénomènes météorologiques et astronomiques. La hauteur d'eau moyenne à la côte par rapport au niveau de référence comprend: l'élévation du niveau de la mer, le niveau de la marée, la surcote atmosphérique créée par le vent qui pousse les masses $d^{\prime}$ 'eau vers la côte et à la dépression qui a un effet de baromètre inverse, la surcote due aux vagues ou set-up qui est une élévation locale du plan d'eau vers la côte due au déferlement des vagues (Fig. 3). En plus de ce niveau moyen s'ajoute le jet de rive ou swash (masses d'eau projetées vers le haut de l'estran par le déferlement des vagues), (Pedreros et al. 2010). Bien que le phénomène de surcote soit exceptionnel (en situation de tempêtes) et de courtes durées, il génère une alimentation, certes intermittente, de la lagune de Kélibia par l'eau de mer pas seulement à travers le grau existant, mais par l'ouverture de plusieurs brèches tout au long du cordon littoral qui sépare la lagune de la mer.

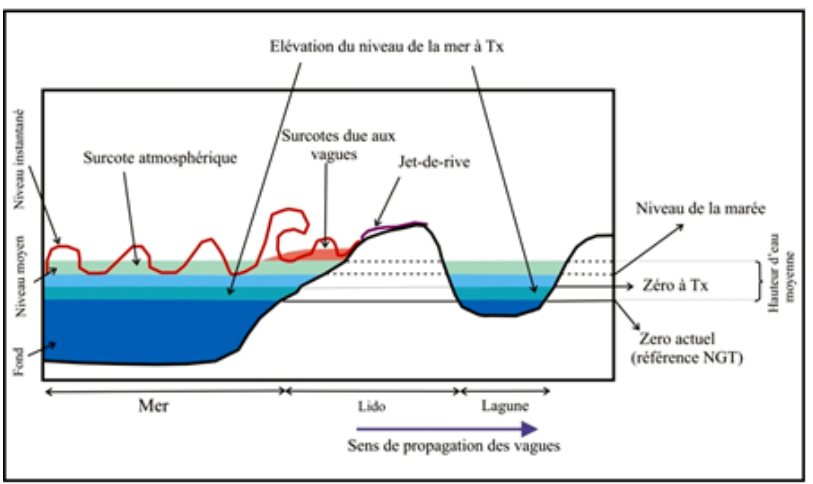

Fig. 2 : Les surfaces inondables dans la lagune côtière de Kẻlibia selon le niveau marin annuel moyen à l'horizon 2100 (Source : Lèves topographiques par GPS; Scénarios de l'IPCC, 1997; IHE, 2000).

La Sebkha de Kélibia comme l'ont constaté plusieurs auteurs sur des littoraux similaires : Durand et Heurtefeux (2006) pour les étangs de Vic et de Pierre Blanche en France, Egis Bceom International/IAU-IDF/BRGM (2009) pour le grand Tunis, Ennesser et al. (2010) pour 
les villes méditerranéennes de l'Afrique du nord; Pedreros et al. (2010) etc., l'ouverture épisodique de brèches lors des tempêtes aurait un impact morphogénique essentiel. En effet, le phénomène d'une accélération de l'élévation du niveau marin et d'un renforcement des tempêtes accroîtraient le risque de morcellement du cordon littoral qui sépare la Sebkha de la mer. En outre, les pertes de matériel sédimentaire pour la plage augmenteront, dans la mesure où, lors d'une tempête, une grande partie des matériaux déplacés par les vagues dans les étangs par submersion ou par ouverture d'une brèche, ne peuvent être récupérées par la suite. Tout ceci constitue un facteur d'accélération du recul de la plage (Fig.3).

En 2100, avec des scénarios d'élévation du niveau marin respectivement de $+38 \mathrm{~cm}$ et $+50 \mathrm{~cm}$ et $55 \mathrm{~cm}$ par rapport à aujourd'hui, les risques de submersion seraient naturellement très importants. La submersion de la lagune serait possible dans les trois scénarios, où le niveau de la mer dépasse la hauteur minimale du cordon littoral, soit plusieurs brèches se produisent dans le lido même pour le scénario volontariste. Nous avons pu localiser sur la figure 3 ces ruptures potentielles. Nous avons considéré que les brèches pouvaient se produire partout dans le cordon littoral et il aurait une fragmentation de ce dernier.

La Sebkhet Kélibia se situe à une côte très basse et un stock dunaire très pauvre. Par une éventuelle élévation accélérée du niveau de la mer, le risque d'érosion est important. Elle serait accompagnée d'une érosion marine menaçante. On s'attendait à disparition de la plage. L'érosion marine sera accélérée et. A la Sebkhet Kélibia, avec une hypothèse d'élévation du niveau de la mer de $0,55 \mathrm{~m}$ à $1^{\prime}$ horizon 2100 , les terres situées à moins de 1 m NGT d'élévation seraient submergées au moins deux fois par an et les terre situées à moins de 1,59 m seraient submergées au moins une fois par 50 an en 2100 .

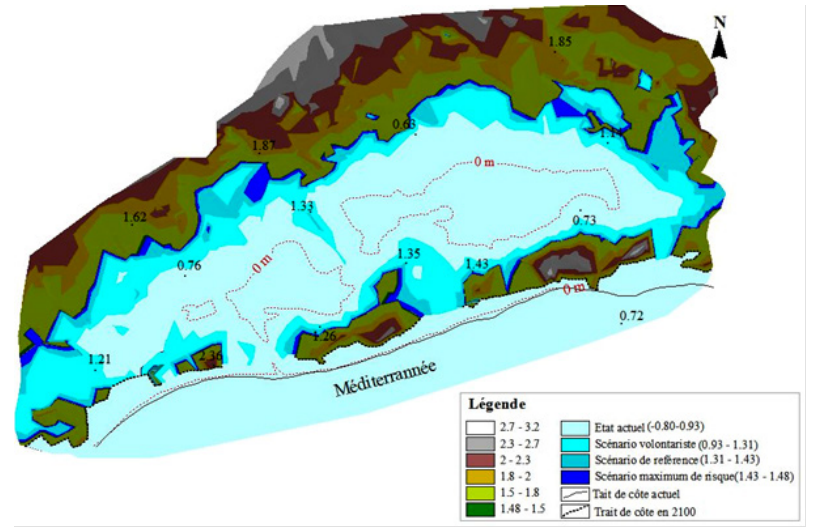

Fig.3. Risque de submersion marine en situation de tempête cinquantennale et évolution future de la lagune de Kélibia.

\section{Impacts environnementaux et socio- économiques de la submersion de la Sebkhet Kélibia}

Pour évaluer les impacts de la submersion marine, il suffit de superposer la carte d'aléa submersion avec la carte des enjeux (humains et économiques...). La cartographie effectuée montre que l'élévation du niveau marin dans la Sebkha de Kélibia pourrait avoir des conséquences importantes. Les plages seront condamnées obligatoirement à disparaître. Dans ce cas, et dans l'impossibilité d'une migration vers le continent, c'est l'érosion qui s'accélèrera et la plage pourra finir par disparaître. L'élévation du niveau marin à la Sebkha de Kélibia serait accompagnée d'une érosion marine menaçante. On s'attendait à l'érosion du cordon dunaire séparant la mer de la sebkha fortement anthropisé. Dans les secteurs fortement aménagés, elle serait accélérée par différentes interventions humaines imprévoyantes (enrochements, murs de protection, épis...etc.). La ville de Kélibia qui a connu, depuis la construction du port au début des années 1980, une extension rapide de l'urbanisation du coté sud du port aux dépens de la lagune subirait les conséquences néfastes de la submersion. Les constructions et les habitations qui sont implantées sur le cordon qui sépare la mer de la sebkha ont généré une perte importante des plages. Ce phénomène risque d'être accentué avec une éventuelle submersion marine et il menace sérieusement toutes les habitations, les constructions hôtelières réalisées sur le cordon existant. La sebkha se transformerait en une lagune. Dans cette conjoncture, et dans l'impossibilité d'une migration, c'est l'érosion qui s'accélèrera et la plage finira par disparaître.

La question du changement climatique s'accompagne inévitablement de problématiques de santé publique. La lagune de Kélibia n'échappe pas à cette règle. La submersion marine entraine la dégradation de la qualité des eaux souterraines et par la même, des systèmes d'évacuations et de filtrations. D'autre part, la modification des écosystèmes a des conséquences sur le cycle d'espèces végétales allergisantes ou les modes de transmission des maladies à transmission vectorielle. En effet, les eaux quasi-stagnantes des marais et des sebkhas sont des hauts lieux de reproduction des moustiques. Les œufs de ces derniers éclosent suite à la chute du taux d'oxygène, provoquée par une immersion (Eche, 2009).

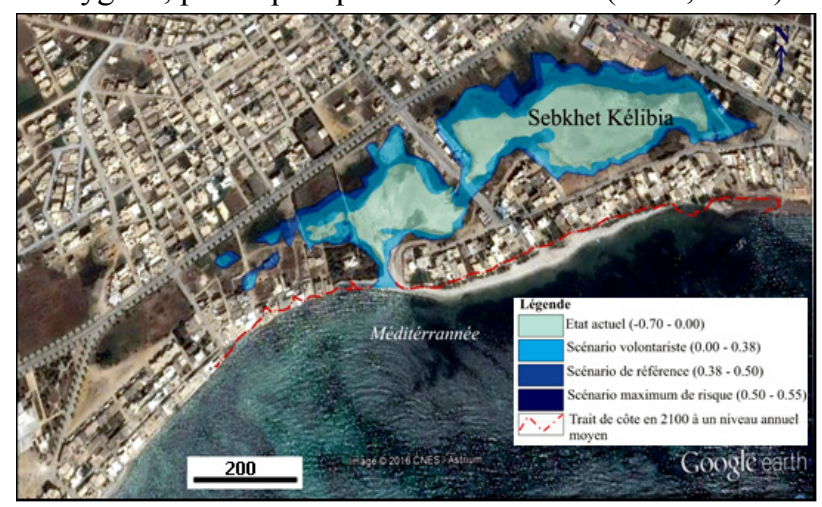

Fig.4 : Le risque de submersion marine à Sebkhet Kélibia en cas d'une élévation du niveau marin par mer moyenne. 


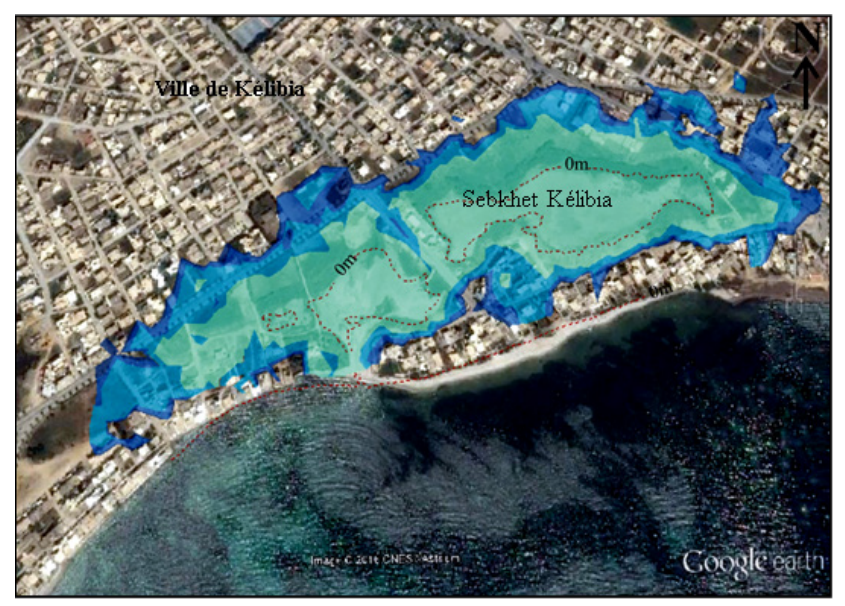

Fig. 5 : Le risque de submersion marine à Sebkhet Kélibia en cas d'une tempête cinquantennale.

\section{Discussion}

La cartographie prévisionnelle du risque submersion marine reste très critiquable puisqu'elle simplifie considérablement un phénomène très complexe, dont on ignore encore pratiquement tout des conséquences morphodyna miques. Toutefois, elle repose sur une hypothèse fondamentale, certes non vérifiable, mais qui correspond à un phénomène déjà observé par plusieurs chercheurs en réponse à une élévation du niveau marin.

\section{Conclusions}

L'étude du risque de submersion marine sur la lagune de Kélibia montre que si l'élévation du niveau marin suit les estimations faites par l'IPCC, les conséquences pourraient devenir catastrophiques pour ce site Ramsar. A l'heure actuelle, le site ne peut pas être submergé dans des conditions normales, c'est-à-dire sans surcote. En 2100, avec des scénarios d'élévation du niveau marin respectivement de $+38 \mathrm{~cm},+50 \mathrm{~cm}$ et $+55 \mathrm{~cm}$ par rapport à aujourd'hui, le risque de submersion seraient naturellement beaucoup plus importants. La submersion de la lagune se traduirait par :

- La disparition de la plage ; dans l'impossibilité d'une migration vers le continent ;

- La submersion des habitations et des constructions hôtelières réalisées sur le cordon littoral ;

- La dégradation de la qualité des eaux souterraines, des systèmes d'évacuations et de filtrations ;

Ainsi, la cartographie des espaces submersibles pourrait être affinée par la réalisation d'une modélisation dynamique fine des épisodes de submersion. En outre, la mise en place d'un suivi morphodynamique s'avérerait très utile pour gérer le risque et conserver l'écosystème. Cependant, la cartographie prévisionnelle du risque submersion marine reste très critiquable puisqu'elle simplifie considérablement un phénomène très complexe, dont on ignore encore pratiquement tout des conséquences morphodynamiques. Cette cartographie étant toutefois à relativiser dans la mesure où les surcotes sont des évènements de fréquence de retour aléatoire.

\section{References}

Anselme B., Durand P., Goeldner-Gianella et Bertrand F. (2008). Impacts de l'élévation du niveau marin sur l'évolution future d'un marais maritime endigue : le domaine de Graveyron, bassin d'Arcachon (France). Vertigo la revue électronique en sciences de l'environnement, vol 8, $\mathrm{N}^{\circ} 1$. http://vertigo.revues.org.

APAL/MEAT (2001) L'hydraulique des zones humides de Maâmoura, Tazarka et Korba, 106 p.

Cariolet J.M.(2011) Inondation des côtes basses et risques associés en Bretagne :Vers une redéfinition des processus hydrodynamiques liés aux conditions météo océaniques et des paramètres morpho-sédimentaires. Thèse de Doctorat en Sciences de la mer, Université de Bretagne Occidentale, $334 \mathrm{p}+$ annexes.

Cariolet J.M, Suanez S., Meur-Férec C. et Postec A. (2012) Cartographie de l'aléa de submersion marine et PPR : éléments de réflexion à partir de l'analyse de la commune de Guissény (Finistère, France). Cybergeo : European Journal of Geography [En ligne], Espace, Société, Territoire, document 586, mis en ligne le 02 février 2012, consulté le 14 février 2017. URL : http://cybergeo.revues.org/25077 ; DOI 10.4000/cybergeo. 25077 .

Durand et Heurtefeux (2006) Impact de l'élévation du niveau marin sur l'évolution future d'un cordon littoral lagunaire: une méthode d'evaluation : Exemple des étangs de Vic et de Pierre Blanche (littoral méditerranéen, France). Annales de Géomorphologie / Annals of Geomorphology / Zeitschrift für Geomorphologie, Schweizerbart und Borntraeger, pp.221-244.

Egis Bceom International/IAU-IDF/BRGM (2011) Adaptation au changement climatique et aux désastres naturels des villes côtières d'Afrique du Nord. Phase 2 : Plan d'adaptation et de résilience - Tunis, Rapport final, $261 p$.

Ennesser Y., Cataliotti D. et Terrier M. (2010) Changement climatique et évaluation des risques côtiers en Afrique du Nord. Journée sur l'impact du changement climatique sur les risques côtiers ; 15-16 novembre 2010 ; BRGM, Orléans, France.

GIEC (2007) : Bilan 2007 des changements climatiques. Contribution des Groupes de travail I, II et III au quatrième Rapport d'évaluation du Groupe d'experts intergouvernemental sur l'évolution du climat [Équipe de rédaction principale, Pachauri, R.K. et Reisinger, A. GIEC, Genève, Suisse, 103 pages.

I.H.E. (2000) Elévation accélérée du niveau de la mer en Tunisie : vulnérabilité et adaptation, Rapport préliminaire 2c, Tunis, $85 \mathrm{p}$.

Pedreros R., Lecacheux S., Delvallée E., Balouin Y., Garcin M., Krien Y., Le Cozannet G., Poisson B. et Thiebot J. (2010) Mise en place d'une approche multimodèles pour évaluer l'exposition du littoral languedocien aux submersions marines dans un contexte de changement climatique (Projet MISEEVA). Journée 
sur l'impact du changement climatique sur les risques côtiers ; 15-16 novembre 2010 ; BRGM, Orléans, France. 